\title{
Fast Parallel Algorithm for Volume Rendering and Its Experiment on Computational Grid ${ }^{\star}$
}

\author{
Hyung-Jun Kim, Sung-Up Jo, Yong-won Kwon, So-Hyun Ryu, \\ Yong-je Woo, Chang-Sung Jeong ${ }^{1}$, and Hyoungwoo Park ${ }^{2}$ \\ 1 Department of Electronics Engineering, Korea University \\ 1-5Ka, Anam-dong, Sungbuk-ku, 136-701, Korea \\ csjeong@charlie.korea.ac.kr \\ 2 Supercomputing Center, KISTI, Daejeon, Korea
}

\begin{abstract}
In this paper we present a fast and well balanced parallel algorithm for volume rendering, and shows our experimental result on Grid computing environment. Our algorithm is based on a new dynamic space leaping method of forward projection, and we shall show that the exploitation of load balancing through the dynamic distribution of data obtained during the execution of the forward projection and the reduction in time taken for the traversal of data and image space sharply speed up the whole algorithm. We implement the parallel ray casting algorithm on computational grid which consists of various heterogenous machines, and have shown that it has relatively good speedup due to the well organized dynamic load balancing.
\end{abstract}

\section{Introduction}

Advances in high speed network and powerful computers make it possible to construct a large-scale high performance distributed computing environments, called a computational grid which uses networks of computers as a single unified computing resource 1]. Computational grid enables large-scale scientific research to better utilize distributed, heterogeneous resources to solve a computational intensive applications by supporting a common set of services and capabilities that are deployed across resources. Globus establishes a software framework for grid infrastructure by providing a meta computer toolkit, called Globus toolkit 2]. In this paper we present a fast and well balanced parallel algorithm for volume rendering, one of the computation intensive problems in visualization area, and shows our experimental result on computational grid using Globus toolkit.

Volume rendering is a technique for visualizing 3D arrays of sampled data. It has applications in various areas such as medical imaging and scientific visualization 3. However, since volume rendering requires high computational costs, the parallel implementation of volume rendering is essential. There have

\footnotetext{
* This work has been supported by KIPA-Information Technology Research Center, University research program by Ministry of Information \& Communication, and Brain Korea 21 projects in 2003
} 
been developed a lot of the parallel techniques developed for volume rendering, but ray casting is one of the volume rendering technique well suited for parallel processing. In ray casting, a ray is casted from the viewpoint through each image pixel and tested for intersection with the volume data. If a ray intersects the volume, the contents of the volume along the ray are sampled, transformed into color and opacity, and composited, and the resulting value is taken as the pixel contents. Ray casting speeds can be improved by a variety of acceleration methods such as adaptive termination [5], adaptive sampling rate along the ray, adaptive refinement, spatial coherency between rays, and space leaping method 8]. In this paper, we are concerned with the design of the parallel ray casting algorithm based on space leaping method.

A number of parallel ray casting algorithms have been reported in the literature 910]. They exploit either image space or object space partition, and each partitioned element is executed as a task in parallel. However, the previous parallel algorithms have some difficulties and limits in load balancing due to the difference of the computation time taken for each ray traversal. In this paper we present a fast and well balanced parallel ray casting algorithm based on dynamic space leaping technique. We shall show that the load balancing by the dynamic distribution of data obtained during the execution of the forward projection and the reduction in time taken for the traversal of data and image space sharply speed up the whole algorithm.

The outline of our paper is as follows: In section 2, we describe basic idea of dynamic space leaping method briefly. In section 3, we give a parallel casting algorithm using dynamic space leaping method, and in section 4, we explain the experimental result which shows the significant speed up of our method. In section 5 , we give a conclusion.

\section{Basic Idea}

One of the most widely used and effective acceleration techniques for ray casting is to efficiently traverse or altogether skip the empty data space and implement re-sampling process only in non-empty data space. This method is called space leaping. In this section we describe a dynamic space leaping method our parallel algorithm is based on.

Space-leaping method skips empty data space to reduce rendering time effectively. However, most of the previous space-leaping methods has three drawbacks which may degrade the whole performance: First, they require a complex ray traversal algorithm in 3D space to skip empty data space or additional 3D memory as large as original volume data to store information on transparent voxels 12,910 . Second, they generate rays for all the pixels in the screen even though some of them are assigned no value since the rays emitted through those pixels do not intersect any objects in data space[6 127]. Third, they has some limit in achieving load balancing for finding non-empty data space or valid image pixels due to the static data distribution[11]. Our dynamic space leaping method overcomes the drawbacks by not only performing efficient skipping pro- 


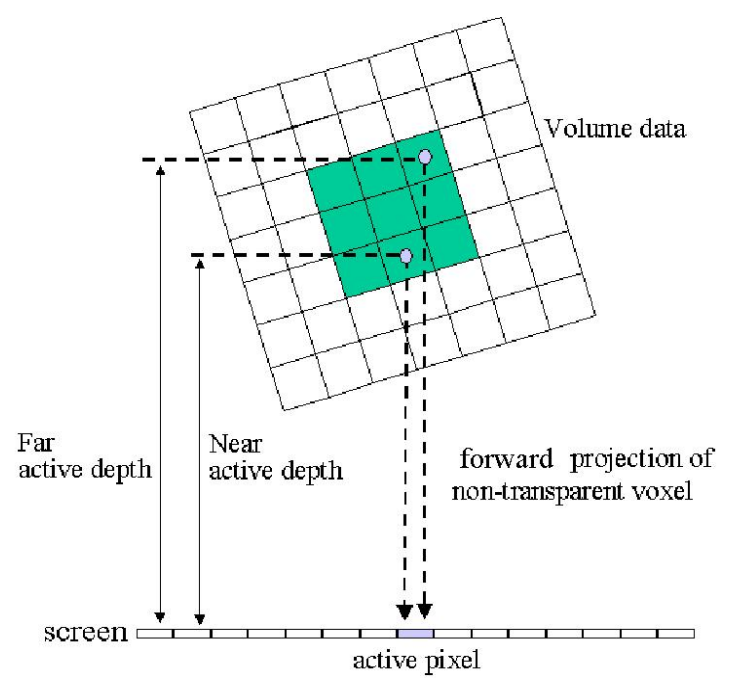

Fig. 1. Forward Projection - Active pixel and active depth

cess in data and image space and but also executing dynamic load balancing for data relevant to the skipping process.

If the ray through a pixel in the screen intersects any non-empty voxel during the traversal through data space, the pixel contributes to the final image, and is called active pixel; otherwise nonactive pixel. Since nonactive pixel does not contribute to the final image and ray does not have to be casted for the nonactive pixel, skipping nonactive pixels in the image space and casting rays only for the active pixels can speed up the whole algorithm in addition to skipping empty space for each ray as in the previous space leaping method.

Dynamic space leaping method not only identifies active pixels, but also stores, in each active pixel, the coordinates of the first and last non-empty voxels encountered by the ray emitted at that pixel. (See figure1,) The first and last non-empty voxels are called nearest and farthest active voxels and their coordinates nearest and farthest active depths respectively. Then, we can start the ray traversal for each active pixel directly from the nearest active depth and stop it at the farthest active depth instead of traversing the entire propagation path. Therefore, we do not need the complex ray traversal algorithm to skip empty space since active depths directly indicate the points where the ray starts and stops the traversal through data space.

The acceleration technique of dynamic space leaping method is based on forward projection which maps each voxel in volume data onto the screen in order to find active pixels and active depths and dynamic distribution of data obtained during forward projection to speed up the calculation of active pixels and active depths as well as the values for each active pixel. During the traversal of volume data, non-empty voxel is projected onto a pixel in the image screen, and the projected pixel is identified as an active pixel, and the coordinate of the 
projected voxel is stored to find the nearest and farthest depths of the pixel. However, traversing all the voxels one by one is not efficient even though it is easy and simple, since it may waste a great amount of time on traversing empty voxels. Our forward projection method makes use of run-length encoded volume data and line drawing algorithm to further improve its speed. By traversing line by line and then slice by slice along volume data, a run-length encoded data is generated which is a series of empty or non-empty voxel runs. By using runlength encoded volume data, we can accelerate the forward projection algorithm by skipping all the empty voxel runs at once. However, it still takes some time to process non-empty voxel run, since we need to traverse each voxel in the non-empty voxel run one by one to project it to the screen. In order to further accelerate the projection process of non-empty voxel run, we exploit line drawing algorithm. For each non-empty voxel run during the traversal of the run-length encoding volume data, its first and last voxels are projected onto the screen, and their corresponding two active pixels are found respectively. Then, the active pixels corresponding to the other voxels of the run are calculated by applying line drawing algorithm to those two active pixels as start and ending pixels respectively. Finally, for each active pixel, its depth to the corresponding voxel is obtained by linear interpolation between the depths of the first and last active pixels. Due to the difference of the length of voxel run and ray traversal path for each active pixel, there are differences in time taken to calculate active pixels and active depths for each voxel run and to find value for each active pixel during the traversal through volume data. In dynamic space leaping method, voxel runs and active pixels obtained during the forward projection are dynamically distributed among processes to achieve load balancing for calculating active pixels and active depths, and their values respectively.

\section{Parallel Volume Rendering}

In this section, we describe a parallel algorithm using the dynamic space leaping method. Our parallel algorithm consists of two phases: calculation of active pixels and active depths for each active pixel, and calculation of values for each active pixel on the screen in the second phase. Our parallel ray casting algorithm achieves the speed up by dynamic distribution of voxel runs for the calculation of active pixels and depths in the line drawing algorithm in the first phase, and the dynamic distribution of active pixels for the calculation of their values through ray traversal in the second phase respectively. In addition, it reduces time taken to traverse in data and image space by skipping empty voxels and non-active pixels respectively during forward projection.

\subsection{Initial Configuration}

Volume rendering requires a large amount of computing power due to its inherent high computational costs. Since Grid supports easy and efficient access 


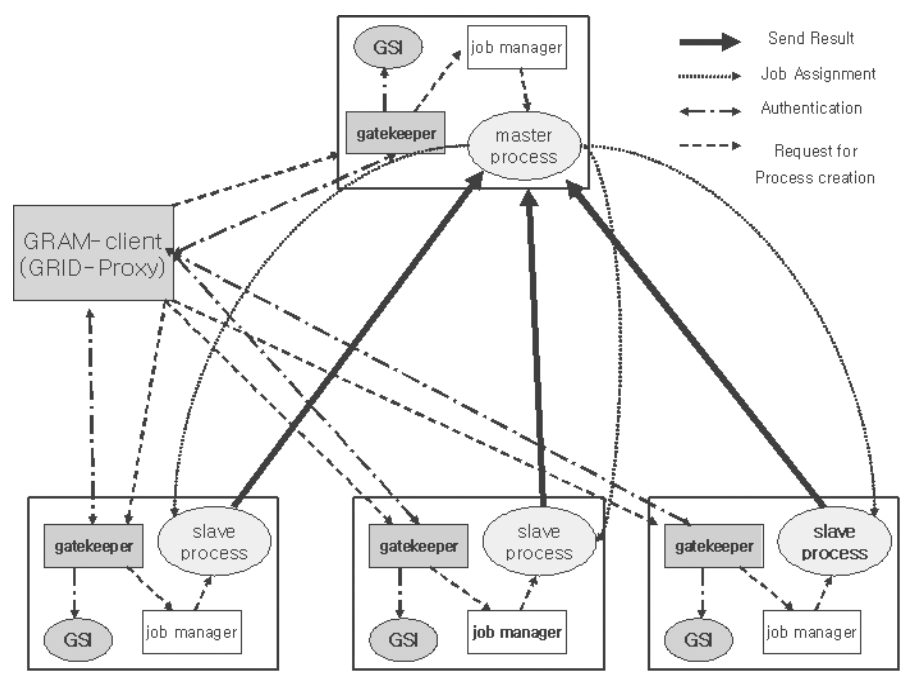

Fig. 2. Master-slave parallel model created by GRAM

to numerous distributed heterogenous computing resources, it provides a computing environment well suited for the design and implementation of our parallel ray casting algorithm. Globus establishes a software framework for Grid infrastructure by providing a meta computer toolkit, called Globus toolkit 2, 1], and becomes a defacto standard for Grid services. In designing and implementing our algorithm, we make use of these technology, including Globus services, such as Resource Management(GRAM)[14, Security Management(GSI), Information Management(MDS) [13] and Data management(Grid FTP) [15], and MPICH-G2 4 which is a message-passing-type communication library based on Globus Toolkit. For the description of our algorithm, we only refer to GRAM and GSI services for simplicity. GRAM(Globus Resource Allocation Manager) consists of gatekeeper and Grid proxy, which are a daemon process on GRID resource node and a client module respectively. GSI(Grid Security Infrastructure) allows a user to authenticate just once per computation, at which time a credential is generated that allows processes created on behalf of user to acquire resources, and so on without additional user intervention.

Figure 2 shows how master and slave processes are created, and how they interact with each other. In Grid, all the participating nodes need a credential issued from CA(Certificated Authorization) so that they can identify one another by mutual authentication. Initial configuration for our parallel ray casting algorithm can be carried out for creation of master and slave processes before executing two phases as follows: First, GRID proxy carries out a mutual authentication with gatekeeper of each allocated node through GSI[16] before sending a request for the creation of process. After mutual authentication, it prepares a job specification using RSL(Resource Specification Language) which describes execution file path, command option and environmental value of the corresponding 


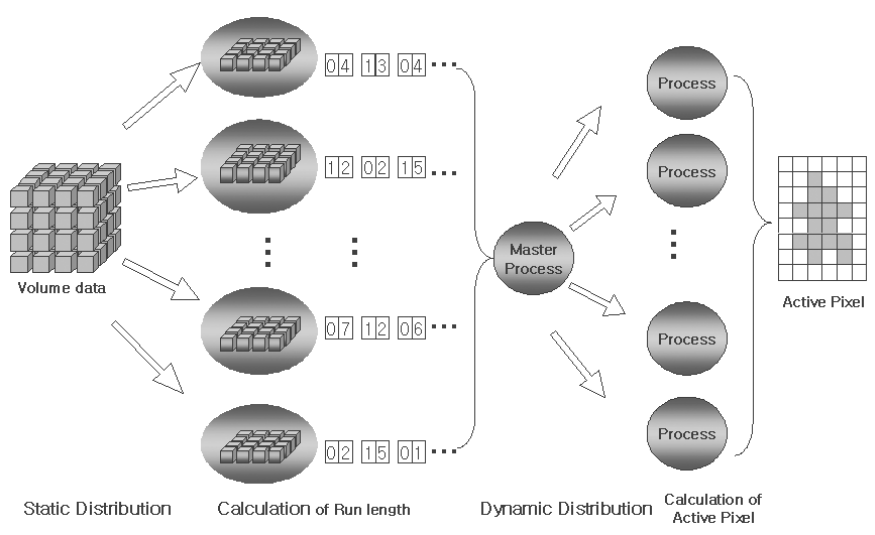

Fig. 3. First Phase

process to be created, and then sends it to a gate-keeper in allocated nodes which in turn activates a local job manager to create one master and slave processes which shall be involved in the computation of our ray casting algorithm. The master and slave processes interact with each other to transfer a job allocation and its result through MPICH-G2[4].

\subsection{First Phase}

In the first phase we find active pixels and active depths by using forward projection as follows: Initially, the volume data is partitioned among allocated processes, and each process finds, for its statically assigned volume data, its runlength encode which consists of non-empty voxel runs. Then, non-empty voxel runs are dynamically distributed among idle processes from the master process, and each process finds active pixels and their active depths for the assigned voxel runs by using line drawing algorithm, and returns them to the master process. Since each computing node has equal amount of load for the calculation of active pixels and depths due to the dynamic distribution of voxel runs, the computation time of active pixels in each process has little difference, and the overall load of the first phase becomes well balanced.

\subsection{Second Phase}

In the second phase, for the ray traversal through volume data, the active pixels obtained in the first phase are distributed among processes (master or slave) from the master process. Here, dynamic load balancing is achieved by continuously supplying idle processes with a small set of active pixels as in the first phase. The value of each active pixel on the screen is calculated as follows: Generate a ray through the active pixel into the data space. Starting at the nearest active depth where the ray intersects non-empty voxel, follow the ray while sampling the 


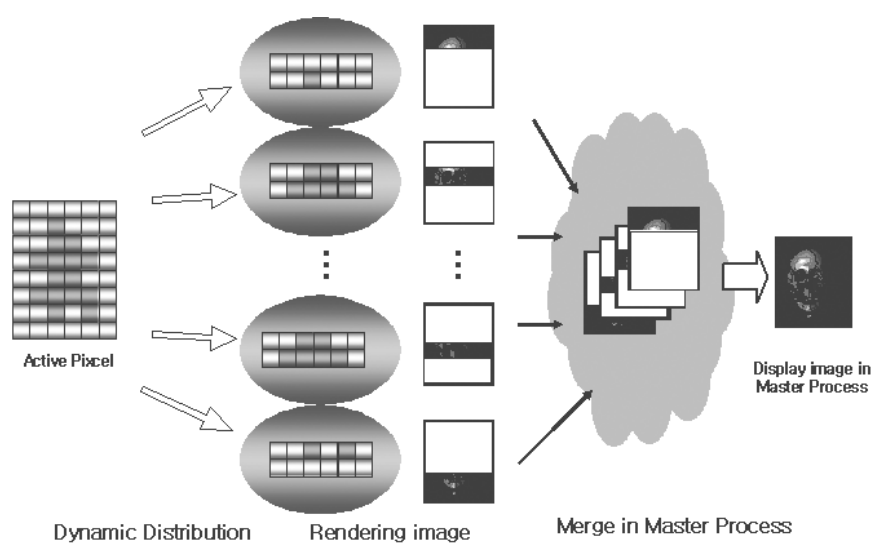

Fig. 4. Second Phase

volume at constant interval. Accumulate the color and opacities of these sampled values. Stop following the ray when it is known that it cannot significantly change its value, or when it intersects the farthest active depth. The resulting partial images are merged to yield the final image in the master process. Similarly as in the first phase, each computing node has equal amount of load for the calculation of values for active pixels due to the dynamic distribution of active pixels, the computation time of values for active pixels in each process has little difference, and the overall load of the second phase becomes well balanced. Also, additional speedup is obtained by performing skipping processes in image and data space based on the information on active pixels and active depths during the ray traversal.

\section{Experiment}

We implemented the parallel ray casting algorithm by using Globus toolkit and MPICH-G2 4 on computational grid environment which consists of 12 heterogeneous machines, two Ultrasparc1, one SGI O2, a SGI Octane, eight Pentium IV PCs connected by 100 Mbps Ethernet. One master process is created on Pentium IV and slave processes on other machines using resource management service in GRAM of Globus toolkit. Our test data set is a $256 \times 256$ x 225 human head, and image screen measures 1024 x 1024 pixels. The details of hardware and software information for each machine are shown in table 1.

Since each machine has different computing power, we have measured the relative performance with $M_{1}$ as reference machine by comparing the execution time of the identical sequential ray casting program on each machine. Then, the expected speed up is computed as a sum of each relative performance of participating machines. The relative performance of the machines obtained by executing the identical sequential ray casting program is shown in table 2. Table 3 shows the execution time, speed up and efficiency of ray casting according 
Table 1. Machine specifications

\begin{tabular}{|c|c|c|c|c|}
\hline Machine type & $M_{1}$ & $M_{2}$ & $M_{3}$ & $M_{4}$ \\
\hline Model & Pentium IV PC & USparc1 & $O_{2}$ & Octane \\
\hline CPU & P IV & UltraSPARC & \multicolumn{2}{|c|}{ MIPS R10000 } \\
\hline Clock(MHz) & 1740 & 143 & 150 & 250 \\
\hline Memory(MBytes) & 1024 & 128 & 128 & 512 \\
\hline OS & Linux 2.2 & Solaris 2.5 & IRIX 6.3 & IRIX 6.5 \\
\hline
\end{tabular}

Table 2. Measurement of relative performance with respect to $M_{1}$ for ray casting

\begin{tabular}{ccccc}
\hline machine $i$ & $M_{1}$ & $M_{2}$ & $M_{3}$ & $M_{4}$ \\
\hline $\begin{array}{c}\text { OS } \\
\text { (spec.) }\end{array}$ & $\begin{array}{c}\text { Linux } \\
(\text { PIV-1.7G) }\end{array}$ & $\begin{array}{c}\text { Solaris2.5 } \\
\text { (USparc1) }\end{array}$ & $\begin{array}{c}\text { IRIX6.3 } \\
\left(O_{2}\right)\end{array}$ & $\begin{array}{c}\text { IRIX6.5 } \\
\text { (Octane) }\end{array}$ \\
\hline running time & 103.01 & 413.812 & 205.390 & 128.690 \\
\hline relative perf. & 1.0 & 0.249 & 0.502 & 0.800 \\
\hline
\end{tabular}

Table 3. Performance results of parallel ray casting on GRID

\begin{tabular}{cccccc}
\hline number of machines & $1\left(M_{1}\right)$ & $2\left(M_{1,4}\right)$ & $4\left(M_{1,2,3,4}\right)$ & $8\left(M_{1,1,1,1,2,2,3,4}\right)$ & $11\left(M_{1,1,1,1,1,1,1,1,2,2,3,4}\right)$ \\
\hline expected speedup & 1.0 & 1.8 & 2.551 & 6.351 & 9.551 \\
\hline \multirow{2}{*}{ GRID } & time (sec) & 103.01 & 69.74 & 49.429 & 20.398 \\
speedup & 1.0 & 1.477 & 2.084 & 5.050 & 7.892 \\
efficiency (\%) & 100.0 & 82.03 & 81.70 & 79.52 & 77.64 \\
\hline
\end{tabular}

to the number of machines. The efficiency represents the ratio of speedup with respect to expected speedup. As the number of machines increases, the parallel algorithm shows relatively good speed up with efficiency around $80 \%$ without degrading its performance due to the communication overhead. This results from the efficient dynamic load balancing from the computation of active pixels and depths and their proper data distribution.

\section{Conclusion}

In this paper we have presented a fast and well balanced parallel algorithm for volume rendering based on the dynamic space leaping method. The acceleration technique of dynamic space leaping method is based on forward projection which allows the fast calculation of active pixels and depths and the dynamic load balancing. We have shown that our parallel ray casting algorithm achieves the speed up by dynamic distribution of voxel runs for the calculation of active pixels and depth in the first phase, and the dynamic distribution of active pixels for the calculation of their values in the second phase respectively. Moreover, additional speed up is obtained by performing skipping processes in image and data space based on the information on active pixels and active depths during the ray traversal in the second phase. We have implemented the parallel ray casting algorithm on computational grid which consists of various heterogenous machines by using Globus toolkit and MPICH-G2, and have shown that it has relatively good speedup due to the well organized dynamic load balancing. 


\section{References}

1. I. Foster, C. Kesselman, S. Tuecke, "The Anatomy of the Grid: Enabling Scalable Virtual Organizations," International J. Supercomputer Applications, 15(3), 2001.

2. I. Foster, and C. Kesselman, "The Globus Project: A Status Report," Heterogeneous Computing Workshop," pp. 4-18, 1998.

3. P. Steven, P. Michael, L. Yarden, S. Peter-Pike, H. Charles, "Interactive Ray Tracing for Volume Visualization," IEEE Trans. on Visualization and Computer Graphics, Vol. 5, No. 3, pp. 238-250. 1999.

4. MPICH-G2, http://www.hpclab.niu.edu/mpi/g2_body.html

5. J. Danskin, and Hanrahan, P., "Fast algorithms for volume ray tracing," 1992 workshop on Volume Visualization, Boston, MA, 1992, pp. 91-98.

6. J. Danskin, R. Bender, and G. T. Herman, "Algebraic reconstruction techniques (ART) for three-dimensional electron microscopy and X-ray photography," J. Theoretical Biology, vol.29, pp. 471-482, 1970.

7. Yagel, R., Cohen, D., Kaufman, A. And Zhang, Q., "Volumetric Ray Tracing," TR 91. 01. 09, Computer Science, SUNY at Stony Brook, January 1991.

8. R. Yagel, and Z. Shi, "Accelerating Volume Animation by Space-Leaping," Visualization '93, 1993, pp. 63-69.

9. V. Goel and A. Mukherjee, "An Optimal Parallel Algorithm for Volume Ray Casting, Visual Comput," Vol. 12, 1996, pp. 26-39.

10. C. Kose and A. Chalmers, "Profiling for efficient parallel volume visualization," Parallel Computing Vol. 23, 1997, pp. 943-952.

11. S. U. Jo and C. S. Jeong, "A Parallel Volume Visualization Using Extended Space Leaping Method," PARA2000, 2001, pp. 296-305.

12. M. Levoy, "A hybrid ray tracer for rendering polygon and volume data," IEEE Computer Graphics \& Application Vol.10, No.2, 1990, pp. 33-40.

13. K. Czajkowski, et al, "Grid Information Services for Distributed Resource Sharing," HPDC-10, August 2001.

14. K. Czajkowski, et al., "Resource Co-Allocation in Computational Grids," HPDC-8, 1999, pp. 219-228.

15. B. Allcock, et al., "Data Management and Transfer in High Performance Computational Grid Environments," Parallel Computing Journal," Vol. 28 (5), May 2002, pp. 749-771.

16. I. Foster, et al. "A Security Architecture for Computational Grids," Proc. 5th ACM CCSC, pp. 83-92, 1998. 